\title{
MÉTODO ALTERNATIVO DE ESTIMACIÓN DEL COEFICIENTE DE VARIACIÓN DE UN EMISOR DE RIEGO LOCALIZADO
}

\author{
Turégano Pastor, J.V. (1) (P), Martí Pérez, P. (2), Royuela Tomás, A. (3)
}

1 Profesor, Universitat Politècnica de València, Departamento de Ingeniería Rural y Agroalimentaria (DIRA), Camino de Vera s/n, 46022 Valencia, jturegan@agf.upv.es

2 Investigador, Institut de Recerca i Tecnologia Agroalimentàries (IRTA), Edifici Fruitcentre, PCiTAL Parc de Gardeny, 25003 Lleida, paumarpe@etsia.upv.es

3 Profesor, Universitat Politècnica de València, Centro Valenciano de Estudios del Riego (CVER), Camino de Vera s/n, 46022 Valencia, aroyuela@agf.upv.es

\begin{abstract}
Resumen
El Coeficiente de Variación de fabricación de un emisor $C V$ se determina según la Norma UNE-EN-ISO-9261 a partir de un único ensayo de laboratorio. Este procedimiento puede conllevar la no correcta determinación del $C V$, ya que en el ensayo los errores de medida atribuibles a los equipos o al proceso de medición pueden provocar la aparición de datos anómalos difíciles de detectar, además de que el CV así obtenido sólo sería válido para la presión de ensayo. En el presente trabajo se utilizan los resultados de los ensayos de 5 emisores no autocompensantes y 5 autocompensantes sometidos a diferentes presiones dentro del rango de trabajo de los mismos, y con ellos se determinan los caudales y los coeficientes de variación reales para cada presión y se ajustan unas funciones de predicción del caudal medio y del caudal emitido por cada emisor individual. A partir de estas funciones, se pueden calcular los caudales y los coeficientes de variación predichos para cada presión. Además, también se proponen unas funciones de ajuste para predecir la evolución del CV con la presión, tanto para los valores de caudal reales como para los predichos. Las principales conclusiones son que el $\mathrm{CV}$ en general depende de la presión para cualquier tipo de emisores y que el modelo propuesto de predicción de los caudales y del $C V$ es correcto y consigue reducir el efecto de los posibles datos de caudal anómalos que aparecen en los ensayos.
\end{abstract}

\section{Introducción y objetivos}

Un emisor de riego localizado es un dispositivo que permite aportar pequeños caudales de agua al suelo, produciendo una gran caída de presión mediante laberintos o a través de toberas u orificios con secciones de paso del agua de muy pequeño tamaño y muy sensibles a la obturación (Keller \& Karmeli, 1974). Como es difícil analizar el régimen hidráulico que se da en el interior de los laberintos, dadas su complejidad y pequeña dimensión, es necesario adoptar un modelo de comportamiento hidráulico para los emisores que permita predecir el caudal arrojado como una función de la presión de entrada al mismo (Demir, Yurdem \& Degirmencioglu, 2007).

El modelo comúnmente adoptado es una ecuación de tipo potencial, como la propuesta por la Norma UNE-EN-ISO 9261 (AENOR, 2010), que relaciona el caudal emitido $q(\mathrm{l} / \mathrm{h})$ con la presión de entrada $h(\mathrm{MPa})$ mediante un coeficiente de descarga $K$ y un exponente $x$ de la siguiente forma: 


$$
\mathrm{q}=\mathrm{K} \cdot \mathrm{h}^{\mathrm{x}}
$$

Esta expresión es válida para emisores no autocompensantes porque refleja muy bien su comportamiento hidráulico real, pero en general no es apropiada para emisores autocompensantes, tal como establecen Royuela y Turégano (2012) y Turégano (2014).

Otro parámetro importante a la hora de definir el comportamiento de los emisores es el denominado Coeficiente de Variación de Fabricación (CV), que es un término usado para describir la variación de los caudales de una muestra de emisores nuevos sometidos a una presión dada (Karmeli \& Keller, 1975).

EI CV se calcula a partir de los caudales recogidos en un ensayo de laboratorio realizado sobre una muestra de emisores y su expresión es:

$$
C V=\frac{\sigma_{q}}{\bar{q}}
$$

Siendo:

- $\sigma_{\mathrm{q}}$ : Desviación típica de los caudales emitidos, en $\mathrm{l} / \mathrm{h}$

- $\bar{q}$ : Promedio de los caudales emitidos, en $1 / h$

El procedimiento que establece la Norma UNE-EN-ISO para la obtención del CV de un emisor consiste en la realización de un único ensayo de laboratorio en el que se somete a una muestra de 25 emisores a su presión nominal si son no autocompensantes y a la presión media del intervalo de compensación si se trata de emisores autocompensantes.

Esta metodología puede no ser adecuada en mucho casos porque:

a) Al realizar solo un ensayo, los errores de medida atribuibles a los equipos o al proceso de medición pueden provocar la aparición de datos anómalos difíciles de detectar y que pueden influir mucho en el valor de CV.

b) Se determina un único valor de $C V$, no teniéndose en cuenta su posible variación con la presión.

Con el presente trabajo se pretende establecer un nuevo método de estimación del $C V$ de un emisor que reduzca, en la medida de lo posible, las incertidumbres asociadas al procedimiento de ensayo y que permita analizar todo el rango de presiones de trabajo del emisor, estableciendo la relación de $C V$ con la presión.

\section{Materiales y métodos}

\subsection{Emisores ensayados}

Se han ensayado 5 emisores no autocompensantes y 5 emisores autocompensantes en el banco de ensayo de emisores del Laboratorio de Riego Localizado del Departamento de Ingeniería Rural y Agroalimentaria de la Universitat Politècnica de València. El procedimiento de ensayo se basó parcialmente en la Norma UNE-EN-ISO 9261 pero fue adaptado a los objetivos y requerimientos del presente trabajo. Se omiten las denominaciones comerciales por razones de confidencialidad.

Así, los emisores no autocompensantes fueron sometidos a cinco niveles de presión distintos: 50, 100, 150, 200 y $250 \mathrm{kPa}$, considerándose su presión nominal $100 \mathrm{kPa}$. 
Por su parte, los emisores autocompensantes fueron sometidos a siete niveles de presión distintos: 100, 150, 200, 250, 300, 350 y $400 \mathrm{kPa}$. En todos los casos se desestimó el nivel propuesto por la norma de $50 \mathrm{kPa}$ al comprobarse que se quedaba fuera del rango de regulación propuesto por los fabricantes y se limitó a $400 \mathrm{kPa}$ como presión máxima que puede soportar una tubería de polietileno según la norma UNE 53367 (AENOR, 2012). Para estos emisores, la presión nominal es la presión media del intervalo de compensación considerado, es decir, $250 \mathrm{kPa}$.

\subsection{Comportamiento hidráulico de los emisores: Predicción del caudal arrojado}

El caudal arrojado es la respuesta de los emisores de riego a la presión de entrada a los mismos. Esta respuesta puede asimilarse a una función matemática que permite predecir el caudal para cualquier presión dada dentro del intervalo de presiones de funcionamiento.

Pero la función matemática, que representa el modelo de comportamiento hidráulico, no puede ser la misma para los emisores no autocompensantes y los autocompensantes. Esto es debido a que los primeros disipan la presión mediante laberintos de largo recorrido o a través de toberas u orificios (Keller \& Karmeli, 1974), mientras que los segundos disponen de un elemento elastomérico móvil cuya geometría y configuración es muy variable (Solomon, 1979) y considerablemente distintas de las de los emisores no autocompensantes.

Por ello, se van a adoptar dos funciones:

a) Función potencial: Para emisores no autocompensantes

b) Función parabólica (polinomio de segundo grado): Para emisores autocompensantes.

La Norma UNE-EN-ISO 9261 (AENOR, 2010) contempla la función potencial para cualquier tipo de emisor, pero la obtiene para el conjunto de los 25 emisores ensayados, es decir, para el caudal medio recogido en cada nivel de presión considerado.

No obstante, dada la previsible variabilidad de los caudales de los diferentes emisores, resulta más interesante obtener las funciones de ajuste para cada emisor individual de manera que se pueda predecir el caudal de cada uno de ellos para cada presión.

Así, las funciones de predicción del caudal, obtenidas mediante ajuste por mínimos cuadrados, son:

Tabla 1: Modelos adoptados para las funciones de predicción del caudal

\begin{tabular}{|c|c|c|}
\hline Tipo de emisor & Caudal individual & Caudal promedio \\
\hline No autocompensante & $\mathrm{q}_{\mathrm{ij}}=\mathrm{K}_{\mathrm{i}} \cdot \mathrm{h}_{\mathrm{j}}^{\mathrm{x}_{\mathrm{i}}}$ & $\overline{\mathrm{q}_{\mathrm{j}}}=\mathrm{K} \cdot \mathrm{h}_{\mathrm{j}}^{\mathrm{x}}$ \\
\hline Autocompensante & $\mathrm{q}_{\mathrm{i}}=\mathrm{A}_{\mathrm{i}}+\mathrm{B}_{\mathrm{i}} \cdot \mathrm{h}_{\mathrm{j}}+\mathrm{C}_{\mathrm{i}} \cdot \mathrm{h}_{\mathrm{j}}^{2}$ & $\overline{\mathrm{q}_{\mathrm{j}}}=\mathrm{A}+\mathrm{B} \cdot \mathrm{h}_{\mathrm{j}}+\mathrm{C} \cdot \mathrm{h}_{\mathrm{j}}^{2}$ \\
\hline
\end{tabular}

Siendo:

- qij: $_{\text {: }}$ Caudal del emisor $i$ sometido a cierta presión $h_{j}$, en $1 / h$

- $\quad \bar{q}_{\mathrm{j}}$ : Media de los caudales de los emisores sometidos a cierta presión $h_{j}$, en $\mathrm{l} / \mathrm{h}$

- $h_{j}$ : Presión de entrada a los emisores, en MPa

- $\mathrm{K}_{\mathrm{i}}, \mathrm{x}_{\mathrm{i}}$ : Coeficiente y exponente de la función potencial de ajuste individual 
- $\mathrm{K}, \mathrm{x}$ : Coeficiente y exponente de la función potencial de ajuste del conjunto de los emisores.

- $A_{i}, B_{i}, C_{i}$ : Coeficientes de la función parabólica de ajuste individual

- A, B, C: Coeficientes de la función parabólica de ajuste del conjunto de emisores

Con este planteamiento se dispone de más información, ya que para cada emisor individual se dispone de dos datos de caudal, uno el realmente obtenido en el ensayo y otro el predicho al aplicar la función de ajuste individual.

\subsection{Obtención del coeficiente de variación de fabricación, $\mathrm{CV}$}

El Coeficiente de Variación de fabricación CV es un indicador de la calidad de fabricación del emisor puesto que recoge la variabilidad relativa de los caudales emitidos por una muestra de emisores del mismo lote sometidos a la misma presión.

La norma UNE-EN-ISO 9261 define un único valor para el CV de un emisor dado, sin tener en cuenta el rango de presiones de trabajo del emisor y su posible influencia en el valor de aquél. Por eso, en el presente trabajo se definen otros $C V$ como alternativa al normativo y que suponen una aproximación más realista a las condiciones de trabajo de un emisor en campo.

Dado que se dispone de los valores de los caudales de todos los emisores para todos los niveles de presión ensayados, es inmediato calcular el CV para cada nivel de presión $h_{j}$.

$$
C V_{j}=\frac{\sigma_{q_{j}}}{\bar{q}_{j}}=\frac{\sqrt{\frac{\sum_{i=1}^{n}\left(q_{i j}-\overline{q_{j}}\right)^{2}}{n-1}}}{\frac{\sum_{i=1}^{n} q_{i j}}{n}}
$$

Donde $n$ es el número de unidades de un mismo emisor ensayadas (es decir, el tamaño de la muestra) y $j$ representa el nivel de presión considerado.

El valor de $q_{i j}$ es el caudal correspondiente a cada unidad de emisor $i$ para la presión dada $h_{j}$, pero como se comentó anteriormente, se puede considerar o bien el caudal realmente recogido en el ensayo o bien el caudal predicho por la función matemática de ajuste que a su vez depende del caudal real. En otras palabras, se pueden definir dos CV distintos según se tomen los caudales reales o los predichos.
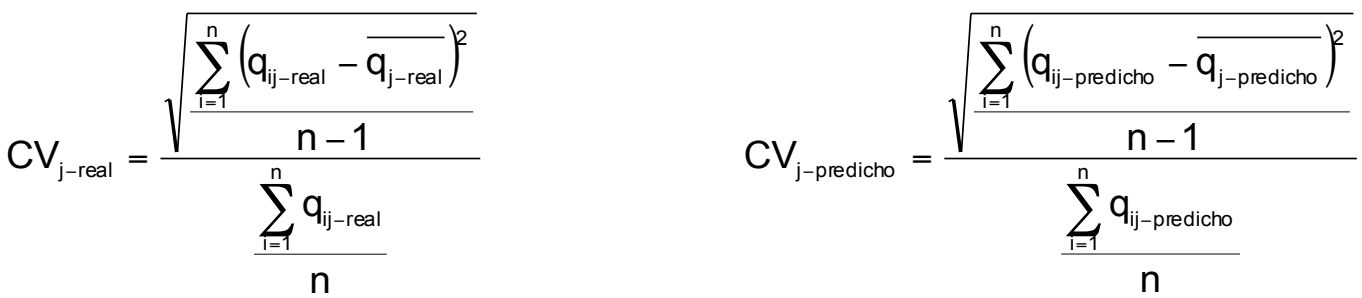

Es de prever que haya diferencias entre estos dos valores para una misma presión, tanto por la incertidumbre inherente al procedimiento de ensayo que puede provocar la aparición de datos anómalos como por la no adecuación de la función de ajuste al comportamiento hidráulico real de los emisores. 
Es decir, suponiendo $p$ niveles de presión $h_{j}$, se dispone de $p$ valores de $C V_{\text {real }}$ y $p$ valores de $C V_{\text {predicho }}$ que permiten apreciar si el Coeficiente de Variación de un emisor varía significativamente con la presión o no. Además, igual que se hace con el caudal de los emisores, también es posible intentar establecer una función de ajuste que relacione el CV con la presión. Con ello, se podría estimar de forma continua el CV para cualquier presión dada dentro del intervalo considerado aunque no fuese una de las presiones de ensayo fijadas. Para ello, se propone buscar una función de ajuste potencial para los emisores no autocompensantes y una función de ajuste parabólica para los emisores no autocompensantes, suponiendo que si el caudal responde a ese tipo de funciones, el CV que se obtiene de este, también responderá a ellas.

Así pues, se definen los $C V_{\text {ensayo }}$ y $C V_{\text {predicho }}$ de la siguiente manera:

Tabla 2: Funciones de ajuste del coeficiente de variación $C V$

\begin{tabular}{|c|c|c|}
\hline Tipo emisor & Datos de ensayo & Datos predichos \\
\hline No autocompensante & $\mathrm{CV}_{\text {ensayo }}=\mathrm{L}_{\text {ensayo }} \cdot \mathrm{h}^{\mathrm{m}_{\text {ensayo }}}$ & $\mathrm{CV}_{\text {predicho }}=\mathrm{L}_{\text {predicho }} \cdot \mathrm{h}^{\mathrm{m}_{\text {preadcho }}}$ \\
\hline Autocompensante & $\mathrm{CV}_{\text {ensayo }}=\mathrm{D}_{\text {ensayo }}+\mathrm{E}_{\text {ensayo }} \cdot \mathrm{h}+\mathrm{F}_{\text {ensayo }} \cdot \mathrm{h}^{2}$ & $\mathrm{CV}_{\text {predicho }}=\mathrm{D}_{\text {predicho }}+\mathrm{E}_{\text {predicho }} \cdot \mathrm{h}+\mathrm{F}_{\text {predicho }} \cdot \mathrm{h}^{2}$ \\
\hline
\end{tabular}

Siendo:

- $L_{\text {ensayo, }} L_{\text {predicho, }} m_{\text {ensayo }}$ y $m_{\text {predicho }}$ los coeficientes y exponentes de las funciones potenciales de ajuste de $C V_{\text {ensayo }}$ y $C V_{\text {predicho, }}$, respectivamente.

- $D_{\text {ensayo }}, E_{\text {ensayo, }} F_{\text {ensayo, }} D_{\text {predicho, }}, E_{\text {predicho }}$ y $F_{\text {predicho }}$ los coeficientes de las funciones parabólicas de ajuste de $C V_{\text {ensayo }}$ y $C V_{\text {predicho, }}$ respectivamente.

Adicionalmente, pueden calcularse otros valores de CV representativos del emisor como son:

a) CV medio para todo el intervalo de presiones de trabajo a partir de los valores discretos:

$$
\overline{\mathrm{CV}}_{\text {discreto }}=\frac{\sum_{\mathrm{j}=1}^{\mathrm{p}} \mathrm{CV}_{\mathrm{j}}}{\mathrm{p}}
$$

b) CV medio para todo el intervalo de presiones de trabajo a partir de la función de ajuste considerada:

$$
\overline{\mathrm{CV}}_{\text {continuo }}=\frac{1}{\mathrm{~h}_{\text {máx }}-\mathrm{h}_{\text {min }}} \int_{\mathrm{h}_{\text {min }}}^{\mathrm{h}_{\text {máx }}} \mathrm{CV}(\mathrm{h}) \cdot \mathrm{dh}
$$

c) CV según la norma UNE-EN-ISO 9261, $\mathrm{CV}_{9261}$ :

$$
\mathrm{CV}_{9261}=\mathrm{CV}\left(\mathrm{h}_{\text {nominal }}\right)
$$




\section{Resultados y discusión}

\subsection{Resultados de los ensayos}

De los ensayos en laboratorio de los 5 emisores no autocompensantes y los 5 emisores autocompensantes se obtienen los siguientes resultados:

- Funciones de predicción del caudal medio.

- Funciones de predicción de los caudales individuales de cada emisor.

- Coeficientes de variación del emisor para cada nivel de presión, tanto para los caudales reales como para los caudales predichos con las funciones individuales.

- Funciones de predicción del Coeficiente de variación del emisor para resultados de ensayo.

- Funciones de predicción del Coeficiente de variación del emisor para los resultados predichos.

- Coeficientes de variación representativos del emisor para los resultados de ensayo.

- Coeficientes de variación representativos del emisor para los resultados predichos.

En algunos emisores se constató la presencia de datos anómalos al realizar una representación de los datos en papel probabilístico normal, por lo que en esos casos el emisor se considera dos veces, la primera con los datos anómalos y la segunda sin ellos. Para distinguirlos se le sumará a la denominación de los primeros la letra "a". Por eso se van a analizar realmente los resultados de 7 casos de emisores no autocompensantes (Noauto1a, Noauto-1, Noauto-2, Noauto-3, Noauto-4, Noauto-5a y Noauto-5) y 9 casos de emisores autocompensantes (Auto-1, Auto-2a, Auto-2, Auto-3a, Auto-3, Auto-4a, Auto-4, Auto-5a y Auto-5)

En la Tabla 3 y la Tabla 4 se dan los coeficientes de ajuste y los coeficientes de correlación al cuadrado tanto para las funciones de ajuste del caudal medio como para las funciones de ajuste del coeficiente de variación, para los emisores no autocompensantes y para los emisores autocompensantes ensayados, respectivamente.

Tabla 3: Funciones de ajuste del caudal medio y el $C V$ para emisores no autocompensantes

\begin{tabular}{|c|c|c|c|c|c|c|c|}
\hline \multirow{2}{*}{ Emisor } & \multicolumn{3}{|c|}{ Ajuste del caudal medio, $q$} & \multicolumn{4}{|c|}{ Ajuste del coeficiente de variación, $C V$} \\
\hline & $\mathbf{K}$ & $\mathbf{x}$ & $\mathbf{R}^{2}$ & & $\mathbf{L}$ & $\mathbf{m}$ & $\mathbf{R}^{2}$ \\
\hline \multirow{2}{*}{ Noauto $1 \mathrm{a}$} & \multirow{2}{*}{12,321} & \multirow{2}{*}{0,4972} & \multirow{2}{*}{0,9998} & Ensayo & 1,481 & $-0,218$ & 0,6114 \\
\hline & & & & Predicho & 1,454 & $-0,232$ & 0,9998 \\
\hline \multirow[b]{2}{*}{ Noauto 1} & \multirow[b]{2}{*}{12,341} & \multirow{2}{*}{0,4956} & \multirow[b]{2}{*}{0,9997} & Ensayo & 1,263 & $-0,115$ & 0,7590 \\
\hline & & & & Predicho & 1,234 & $-0,126$ & 0,9922 \\
\hline \multirow{2}{*}{ Noauto 2} & \multirow{2}{*}{13,298} & \multirow{2}{*}{0,5252} & \multirow{2}{*}{0,9999} & Ensayo & 1,332 & 0,163 & 0,4360 \\
\hline & & & & Predicho & 1,301 & 0,155 & 0,9869 \\
\hline \multirow{2}{*}{ Noauto 3} & \multirow{2}{*}{7,195} & \multirow{2}{*}{0,5096} & \multirow{2}{*}{0,9998} & Ensayo & 2,389 & $-0,021$ & 0,7105 \\
\hline & & & & Predicho & 2,389 & $-0,021$ & 0,9995 \\
\hline \multirow{2}{*}{ Noauto 4} & \multirow{2}{*}{12,817} & \multirow{2}{*}{0,5095} & \multirow{2}{*}{0,9998} & Ensayo & 1,937 & $-0,009$ & 0,4676 \\
\hline & & & & Predicho & 1,935 & $-0,009$ & 0,9890 \\
\hline \multirow{2}{*}{ Noauto $5 a$} & \multirow{2}{*}{6,945} & \multirow{2}{*}{0,5326} & \multirow{2}{*}{1,0000} & Ensayo & 2,638 & $-0,164$ & 0,1982 \\
\hline & & & & Predicho & 2,482 & $-0,204$ & 0,9988 \\
\hline \multirow{2}{*}{ Noauto 5} & \multirow{2}{*}{6,948} & \multirow{2}{*}{0,5305} & \multirow{2}{*}{0,9999} & Ensayo & 2,975 & 0,035 & 0,0762 \\
\hline & & & & Predicho & 2,964 & 0,032 & 0,9132 \\
\hline
\end{tabular}


Tabla 4: Funciones de ajuste del caudal medio y el $C V$ para emisores autocompensantes

\begin{tabular}{|c|c|c|c|c|c|c|c|c|c|}
\hline \multirow{2}{*}{ Emisor } & \multicolumn{4}{|c|}{ Ajuste del caudal medio, $q$} & \multicolumn{5}{|c|}{ Ajuste del coeficiente de variación, $C V$} \\
\hline & A & B & C & $\mathbf{R}^{2}$ & & D & E & $\mathbf{F}$ & $\mathbf{R}^{2}$ \\
\hline \multirow{2}{*}{ Auto 1} & \multirow{2}{*}{2,404} & \multirow{2}{*}{$-1,634$} & \multirow{2}{*}{3,243} & \multirow{2}{*}{0,84} & Ensayo & 2,613 & $-6,459$ & 15,055 & 0,69 \\
\hline & & & & & Predicho & 3,105 & $-11,579$ & 25,442 & 0,93 \\
\hline \multirow{2}{*}{ Auto 2a } & \multirow{2}{*}{3,499} & \multirow{2}{*}{10,123} & \multirow{2}{*}{$-17,567$} & \multirow{2}{*}{0,95} & Ensayo & 20,747 & $-93,714$ & 143,61 & 0,98 \\
\hline & & & & & Predicho & 21,752 & $-104,95$ & 166,25 & 1,00 \\
\hline \multirow[b]{2}{*}{ Auto 2} & \multirow[b]{2}{*}{3,620} & \multirow[b]{2}{*}{9,863} & \multirow{2}{*}{$-17,315$} & \multirow{2}{*}{0,95} & Ensayo & 17,785 & $-80,622$ & 119,18 & 0,99 \\
\hline & & & & & Predicho & 17,653 & $-80,236$ & 118,72 & 1,00 \\
\hline \multirow{2}{*}{ Auto $3 a$} & \multirow{2}{*}{3,871} & \multirow{2}{*}{$-0,124$} & \multirow{2}{*}{2,768} & \multirow{2}{*}{0,87} & Ensayo & 2,135 & $-7,942$ & 23,884 & 0,89 \\
\hline & & & & & Predicho & 1,993 & $-5,485$ & 17,433 & 0,98 \\
\hline \multirow{2}{*}{ Auto 3} & \multirow{2}{*}{3,829} & \multirow{2}{*}{$-0,189$} & \multirow{2}{*}{3,091} & \multirow{2}{*}{0,95} & Ensayo & 1,667 & $-2,507$ & 12,175 & 0,90 \\
\hline & & & & & Predicho & 1,697 & $-3,096$ & 13,500 & 0,97 \\
\hline \multirow{2}{*}{ Auto 4a } & \multirow{2}{*}{2,081} & \multirow{2}{*}{$-0,139$} & \multirow{2}{*}{1,172} & \multirow{2}{*}{0,89} & Ensayo & 2,964 & 0,792 & $-2,529$ & 0,15 \\
\hline & & & & & Predicho & 4,078 & $-6,494$ & 8,354 & 0,97 \\
\hline \multirow{2}{*}{ Auto 4} & 2051 & R & 1520 & 0 & Ensayo & 1,991 & $-2,345$ & 9,509 & 0,79 \\
\hline & ו I & -u, & 1 & ט, & Predicho & 2,307 & $-5,356$ & 15,221 & 0,89 \\
\hline Auto $5 a$ & 3.705 & -3.223 & 6094 & 089 & Ensayo & 1,009 & 6,092 & $-7,624$ & 0,46 \\
\hline Auto od & 3,105 & $-3,<<0$ & 0,094 & 0,09 & Predicho & 2,204 & $-7,312$ & 20,282 & 0,90 \\
\hline Auto 5 & 3,700 & $-3,141$ & 5,912 & 0.92 & Ensayo & 1,523 & $-1,616$ & 9,502 & 0,82 \\
\hline Auto 5 & 0,100 & $-3,141$ & 0,912 & 0,92 & Predicho & 2,462 & $-11,638$ & 29,884 & 0,88 \\
\hline
\end{tabular}

Tabla 5: Funciones de ajuste de los caudales de los emisores individuales. Ejemplo de emisor no autocompensante (izda.) y emisor autocompensante (dcha.)

\begin{tabular}{|c|c|c|c|c|c|c|c|c|}
\hline \multicolumn{4}{|c|}{ No autocompensante (Noauto-1a) } & \multicolumn{5}{|c|}{ Autocompensante (Auto-3a) } \\
\hline Emisor & $\mathrm{K}_{\mathrm{i}}$ & $\mathbf{x}_{\mathbf{i}}$ & $\mathbf{R}^{2}$ & Emisor & $A_{i}$ & $B_{i}$ & $\mathrm{C}_{\mathrm{i}}$ & $\mathbf{R}^{2}$ \\
\hline 1 & 12,488 & 0,495 & 0,9997 & 1 & 3,859 & 0,173 & 2,706 & 0,8705 \\
\hline 2 & 12,140 & 0,495 & 0,9997 & 2 & 3,966 & $-0,181$ & 2,502 & 0,8171 \\
\hline 3 & 12,378 & 0,496 & 0,9997 & 3 & 3,880 & $-1,098$ & 5,488 & 0,9449 \\
\hline 4 & 12,377 & 0,496 & 0,9997 & 4 & 3,996 & $-0,589$ & 3,928 & 0,7337 \\
\hline 5 & 12,199 & 0,493 & 0,9996 & 5 & 3,780 & $-0,287$ & 2,229 & 0,7846 \\
\hline 6 & 12,531 & 0,497 & 0,9997 & 6 & 3,930 & $-0,372$ & 3,105 & 0,8647 \\
\hline 7 & 12,271 & 0,495 & 0,9997 & 7 & 3,869 & 0,197 & 2,281 & 0,8836 \\
\hline 8 & 12,141 & 0,501 & 0,9998 & 8 & 3,679 & 0,964 & 0,352 & 0,6113 \\
\hline 9 & 11,898 & 0,513 & 0,9970 & 9 & 3,773 & 0,822 & 1,084 & 0,9021 \\
\hline 10 & 12,054 & 0,486 & 0,9994 & 10 & 3,952 & $-0,876$ & 3,766 & 0,8562 \\
\hline 11 & 12,208 & 0,496 & 0,9997 & 11 & 3,735 & 0,625 & 1,452 & 0,8376 \\
\hline 12 & 12,524 & 0,495 & 0,9997 & 12 & 3,790 & 0,644 & 2,046 & 0,9101 \\
\hline 13 & 12,210 & 0,495 & 0,9997 & 13 & 3,899 & 0,002 & 2,607 & 0,8618 \\
\hline 14 & 12,277 & 0,495 & 0,9997 & 14 & 3,956 & $-0,707$ & 4,234 & 0,8998 \\
\hline 15 & 12,682 & 0,495 & 0,9998 & 15 & 3,877 & 0,155 & 2,348 & 0,9152 \\
\hline 16 & 12,402 & 0,495 & 0,9997 & 16 & 3,865 & 0,015 & 2,719 & 0,9095 \\
\hline 17 & 12,489 & 0,493 & 0,9997 & 17 & 3,795 & $-0,120$ & 2,469 & 0,8423 \\
\hline 18 & 12,201 & 0,491 & 0,9996 & 18 & 3,998 & $-0,853$ & 3,638 & 0,8134 \\
\hline 19 & 12,370 & 0,498 & 0,9997 & 19 & 3,973 & $-0,411$ & 3,746 & 0,8779 \\
\hline 20 & 12,628 & 0,492 & 0,9997 & 20 & 3,750 & 1,045 & 1,302 & 0,8547 \\
\hline 21 & 12,143 & 0,495 & 0,9998 & 21 & 4,045 & $-1,507$ & 3,503 & 0,4059 \\
\hline 22 & 12,617 & 0,500 & 0,9996 & 22 & 4,040 & $-0,918$ & 3,116 & 0,8668 \\
\hline 23 & 12,178 & 0,494 & 0,9997 & 23 & 3,925 & $-0,862$ & 3,783 & 0,8054 \\
\hline 24 & 12,351 & 0,511 & 0,9999 & 24 & 3,727 & 0,699 & 2,128 & 0,9251 \\
\hline 25 & 12,297 & 0,521 & 0,9995 & 25 & 3,714 & 0,332 & 2,657 & 0,9676 \\
\hline Promedio & 12,322 & 0,497 & & Promedio & 3,871 & $-0,124$ & 2,768 & \\
\hline
\end{tabular}




\subsection{Funciones de predicción del caudal de los emisores}

\subsubsection{Emisores no autocompensantes}

A la vista de los resultados expuestos en la Tabla 3, se comprueba que el modelo de función potencial adoptado es perfectamente válido para todos los casos. Es decir, explica la práctica totalidad de los datos medios observados al obtenerse $\mathrm{R}^{2}$ muy próximos a la unidad.

De manera análoga, se pueden hacer ajustes individuales para cada elemento emisor ensayado, obteniendo tantas funciones de ajuste como emisores componen la muestra.

Analizado los resultados obtenidos expuestos en la Tabla 5 se observa que dichos ajustes individuales también son muy buenos, en virtud de los coeficientes de correlación $R^{2}$ calculados. No obstante, se aprecian ciertas diferencias entre los valores de los coeficientes $K_{i}$ y los exponentes $x_{i}$ de los diferentes emisores individuales, que son precisamente las que originan la variabilidad de los caudales emitidos. Esto contrasta con lo expuesto por Bralts et al. (1981) quienes consideraban que la variabilidad de los emisores debida al proceso de fabricación se podía asignar completamente a la variabilidad del coeficiente $K_{i}$ dado que el exponente $x$ se mantendría constante.

También se constata que el valor medio de $x_{i}$ coincide exactamente con el valor de $x$ para el ajuste del caudal medio pero el valor medio de $K_{i}$ difiere levemente del valor de $K$ para el ajuste del caudal medio, tal como demostró Turégano (2014).

\subsubsection{Emisores no autocompensantes}

En este tipo de emisores el modelo de comportamiento hidráulico consiste en una función polinómica de grado 2 (función parabólica). Los resultados obtenidos para las funciones de ajuste del caudal medio con la presión, figuran en la Tabla 4.

Atendiendo a los valores de $R^{2}$ el modelo parabólico adoptado para los emisores autocompensantes no predice tan bien el caudal emitido como lo hacía el modelo potencial para los emisores no autocompensantes. No obstante, la función parabólica se ajusta mucho mejor que la función potencial para los emisores autocompensantes.

En cuanto a los ajustes individuales de cada unidad emisora (Ver Tabla 5), se demuestra que el modelo parabólico también es apropiado aunque hay gran variabilidad en los diferentes valores de $A_{i}, B_{i}$ y $C_{i}$.

Por otra parte, se constata que los promedios de $A_{i}, B_{i}$ y $C_{i}$ coinciden exactamente con los valores de $A, B$ y $C$ obtenidos para el ajuste de los caudales medios, corroborando lo expuesto por Turégano (2014).

\subsection{Coeficientes de variación de fabricación, $C V$}

\subsubsection{Variación con la presión}

En general se observa que el coeficiente de variación de fabricación $C V$ en las dos formas propuestas (ensayo y predicho) varía con la presión, tanto para emisores no autocompensantes como autocompensantes. Esto contrasta con la establecido por Bralts et al. (1981) o por Rodrigo et al. (1997). Además, la mayor parte de las propuestas que tienen 
relación con el cálculo de la uniformidad de distribución en sus diferentes formatos o con el diseño hidráulico de subunidades, se basan en la constancia de dicho parámetro (Karmeli y Keller 1975, Bralts et al. 1987, Burt et al. 1997, Ascough y Kiker 2002, Barragan et al. 2006, Noori y Al Thamiry 2012, Zhang et al. 2013).

Si se analizan los valores concretos del CV para cada una de las presiones de ensayo, se aprecia cierta diferencia entre los valores procedentes del propio ensayo y los valores predichos. Esto en principio no permitiría establecer una tendencia de variación del $C V$ con la presión, al menos para los $C V$ de ensayo. Pero si se modeliza dicha variación mediante una función potencial para los emisores no autocompensantes o una función parabólica para los emisores autocompensantes sí que es posible predecir la citada tendencia.

Así, a la vista de los resultados expuestos en la Tabla 3, la Tabla 4 y la Figura 1, se aprecia que las funciones de ajuste de los $C V_{\text {predicho }}$ presentan valores de $R^{2}$ claramente superiores a las de las funciones de ajuste de los $C V_{\text {ensayo. }}$ Esto se debe a que los errores de medida inherentes a los procedimientos y métodos de ensayo no se manifiestan en el cálculo del $C V_{\text {predicho, }}$ que se realiza a partir de las funciones de ajuste individuales de los emisores.

No obstante, la representación gráfica de las funciones de ajuste pone de manifiesto que las curvas correspondientes a los $C V_{\text {predicho y }} C V_{\text {ensayo }}$ son prácticamente coincidentes (Ver Figura 1), a pesar de que el coeficiente $L$ y el exponente $m$ para la función potencial y los coeficientes $D, E$ y $F$ para la función parabólica en uno y otro caso aparentemente difieren bastante.
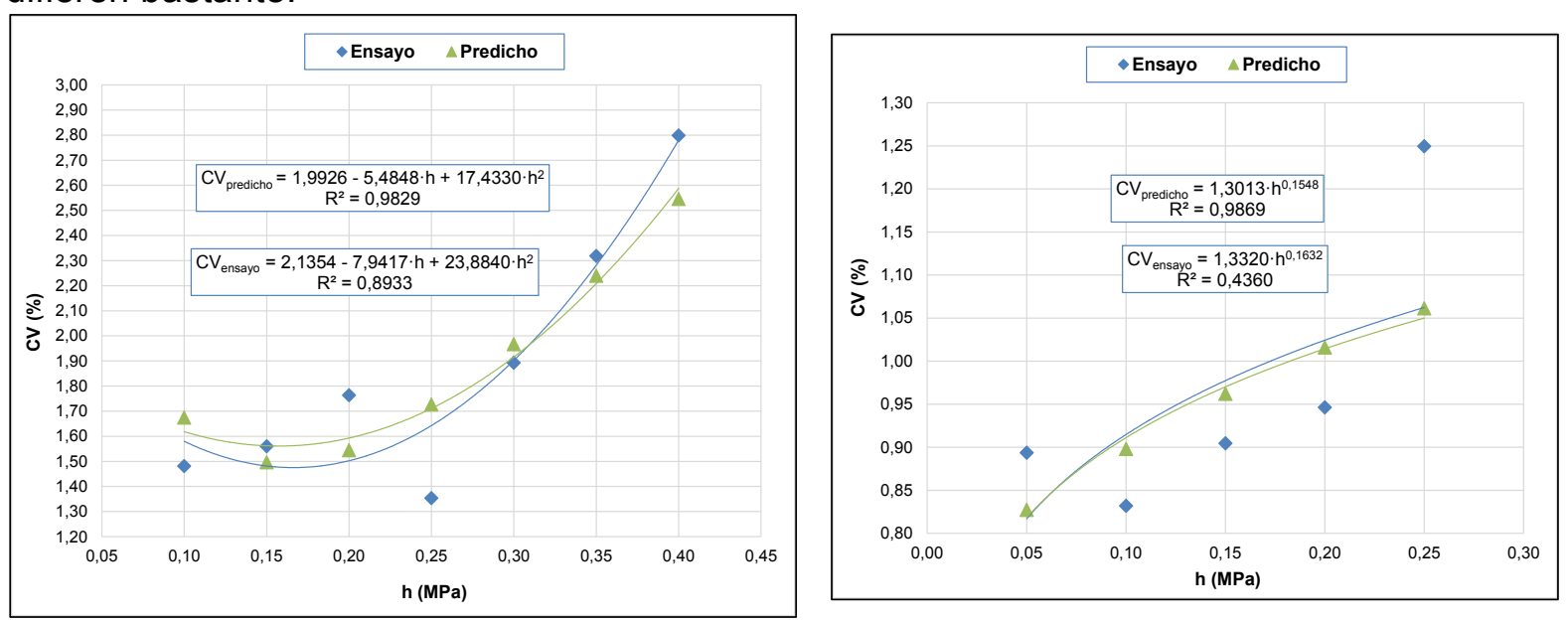

Figura 1: Ejemplo de variación de $C V$ con la presión para el emisor autocompensante Auto-3a (izda.) y el emisor no aucompensante Noauto-2(dcha.)

Por otra parte, en el caso de los emisores autocompensantes, se comprueba que los mejores ajustes para las funciones parabólicas del CV se dan cuando los ajustes de las funciones parabólicas para el caudal también son los mejores. Esto puede constatarse por ejemplo en el caso del emisor Auto 2a que tiene un $R^{2}$ para el ajuste del $C V$ de 0,9781 y un $R^{2}$ para el ajuste del caudal de 0,9471 . En definitiva, cuanto mejor explica el modelo parabólico el caudal emitido, mejor explica el modelo parabólico la evolución del CV con la presión. 


\subsubsection{Comparativa entre los diferentes $C V$ representativos del emisor}

En la tabla siguiente se incluyen todos los CV representativos de un emisor obtenidos de la forma expuesta en la sección Materiales y métodos tanto para los resultados de los ensayos como para los caudales predichos según las funciones de ajuste individuales de los emisores.

Tabla 6: Coeficientes de variación representativos de los emisores

\begin{tabular}{|c|c|c|c|c|c|c|c|}
\hline \multicolumn{2}{|c}{ Emisor } & \multicolumn{2}{c|}{$\overline{\mathbf{C V}}_{\text {continuo }}$} & \multicolumn{2}{c|}{$\overline{\mathbf{C V}}_{\text {discreto }}$} & \multicolumn{2}{c|}{ CV $_{\text {9261 }}$} \\
\cline { 3 - 8 } & Ensayo & Predicho & Ensayo & Predicho & Ensayo & Predicho \\
\hline \multirow{5}{*}{$\begin{array}{c}\text { No } \\
\text { autocomp. }\end{array}$} & Noauto 1a & 2,293 & 2,315 & 2,340 & 2,353 & 2,447 & 2,480 \\
\cline { 2 - 8 } & Noauto 1 & 1,587 & 1,588 & 1,602 & 1,598 & 1,649 & 1,645 \\
\cline { 2 - 8 } & Noauto 2 & 0,966 & 0,959 & 0,965 & 0,953 & 0,915 & 0,911 \\
\cline { 2 - 8 } & Noauto 3 & 2,491 & 2,491 & 2,494 & 2,493 & 2,507 & 2,508 \\
\cline { 2 - 8 } & Noauto 4 & 1,972 & 1,970 & 1,974 & 1,973 & 1,978 & 1,976 \\
\cline { 2 - 8 } & Noauto 5a & 3,662 & 3,735 & 3,855 & 3,915 & 3,848 & 3,970 \\
\cline { 2 - 8 } & Noauto 5 & 2,777 & 2,783 & 2,761 & 2,764 & 2,746 & 2,755 \\
\hline \multirow{5}{*}{ Autocomp. } & Auto 1 & 2,052 & 1,991 & 2,090 & 2,055 & 1,939 & 1,801 \\
\cline { 2 - 8 } & Auto 2a & 7,371 & 7,153 & 7,730 & 7,568 & 6,294 & 5,906 \\
\cline { 2 - 8 } & Auto 2 & 5,972 & 5,904 & 6,270 & 6,201 & 5,078 & 5,014 \\
\cline { 2 - 8 } & Auto 3a & 1,822 & 1,842 & 1,882 & 1,885 & 1,643 & 1,711 \\
\cline { 2 - 8 } & Auto 3 & 1,893 & 1,868 & 1,923 & 1,902 & 1,801 & 1,767 \\
\cline { 2 - 8 } & Auto 4a & 2,985 & 3,039 & 2,978 & 3,060 & 3,004 & 2,977 \\
\cline { 2 - 8 } & Auto 4 & 2,070 & 2,033 & 2,094 & 2,071 & 1,999 & 1,919 \\
\cline { 2 - 8 } & Auto 5a & 1,998 & 1,796 & 1,979 & 1,846 & 2,055 & 1,643 \\
\cline { 2 - 8 } & Auto 5 & 1,784 & 1,644 & 1,808 & 1,719 & 1,713 & 1,420 \\
\hline
\end{tabular}

En casi todos los casos se observa muy poca diferencia entre los valores de CV obtenidos del ensayo u obtenidos a partir de la predicción de los caudales. Esto significa que la consideración de las funciones de ajuste individuales de cada emisor, utilizadas para calcular los caudales predichos, también permite predecir el $C V$ con fiabilidad.

Comparando los valores medios, $\overline{C V}_{\text {continuo }}$ y $\overline{C V}_{\text {discreto }}$ en el rango de presiones considerado, se aprecia poca diferencia para la mayoría de los emisores, tanto no autocompensantes como autocompensantes (excepto para el Noauto 5a, Auto 2a y Auto 2). Teniendo en cuenta que el $\overline{C V}_{\text {continuo }}$ se calcula utilizando la función de ajuste del $C V$ con la presión y que el $\overline{C V}_{\text {discreto }}$ se calcula con los valores de $C V$ para cada nivel de presión de ensayo, se puede colegir que el modelo propuesto para predecir la variación del CV con la presión es adecuado.

En lo que respecta al $C V_{9261}$ (que se ha calculado a partir de las funciones de ajuste del $C V$ propuestas), se ve que en general difiere de los valores medios antes mencionados, siendo esta diferencia mucho más acusada para los emisores autocompensantes. La influencia de estas desviaciones no es importante para los emisores no autocompensantes, ya que las subunidades de las que forman parte se suelen diseñar para que la presión media sea la nominal y las variaciones relativas de presión no excedan del $20 \%$, pero sí que lo es para los autocompensantes, puesto que las subunidades constituidas por ellos se $p$ de manera que se cubra todo el intervalo de presiones de compensación por lo que el $C V_{9261}$ no sería la referencia adecuada para establecer el CV representativo del emisor. 


\subsubsection{Influencia de la presencia de datos anómalos}

Como se puede ver en la Figura 2, la presencia de emisores con datos de caudal anómalos hace que en general el CV sea más elevado ya que dichos datos modifican sustancialmente el valor de las desviaciones típicas. Además, también provoca mayores diferencias entre los valores predichos y los valores de ensayo.

Al rehacer los cálculos sin los emisores con datos anómalos, se observa una mayor modificación de los $C V$ de ensayo (tanto de los valores observados para cada nivel de presión como para la función de ajuste parabólica correspondiente), que de los CV obtenidos con los caudales predichos. Por tanto, en los casos en que sea difícil detectar datos anómalos, es mejor utilizar los valores predichos de caudal individual de cada emisor para determinar el coeficiente de variación $C V_{\text {predicho }}$ así como su función de ajuste, porque se reduce la influencia de aquéllos.
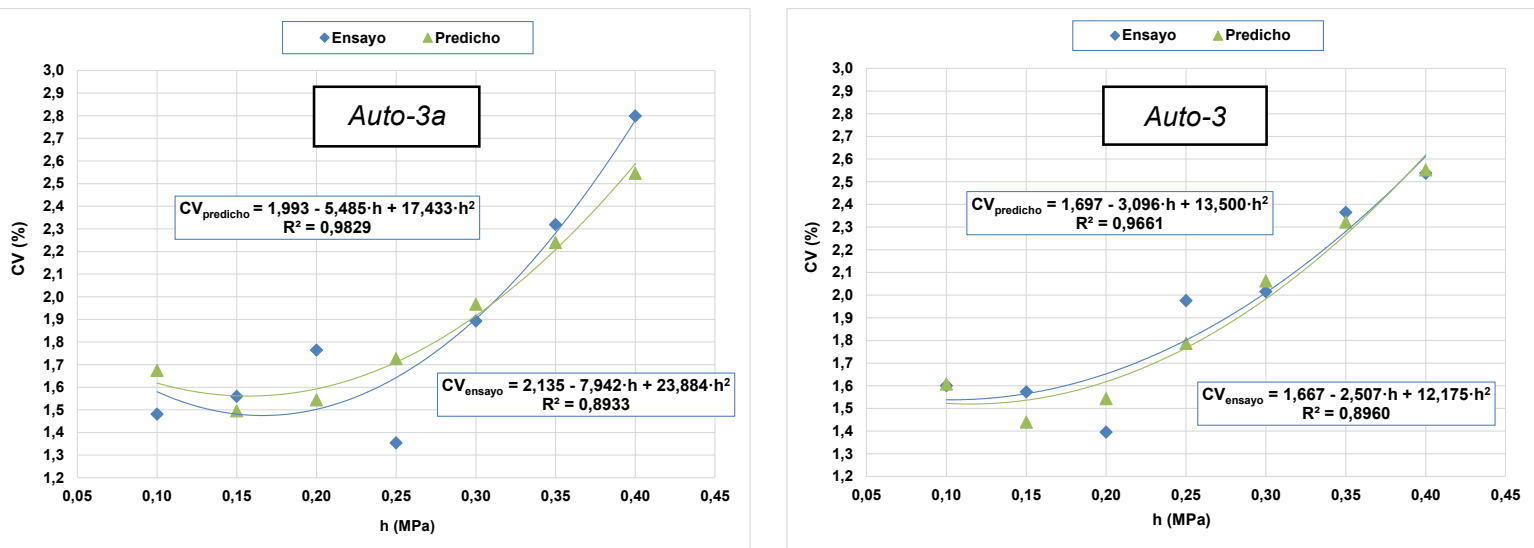

Figura 2: Ejemplo de funciones de ajuste del $C V$ para un emisor autocompesante. Con datos anómalos (izda.) y sin datos anómalos (dcha.)

\section{Conclusiones y recomendaciones}

A la vista de los resultados, es posible enumerar las siguientes conclusiones:

a) La función potencial de ajuste es un buen modelo de predicción del caudal emitido para los emisores no autocompensantes pero no para los autocompensantes en los que el mejor modelo de predicción del caudal emitido es el de la función parabólica. Esto se cumple tanto para el caudal medio de una muestra de emisores como para el caudal individual de cada elemento.

b) Para los emisores no autocompensantes, el coeficiente $K$ de ajuste de la función potencial de predicción del caudal medio difiere ligeramente del promedio de los coeficientes $K_{i}$ de ajuste del caudal de cada emisor individual. El exponente $x$ de ajuste para la función potencial de predicción del caudal medio es exactamente igual que el promedio de los exponentes $x_{i}$ de ajuste del caudal de cada emisor individual.

c) Para los emisores autocompensantes, los coeficientes $A, B$ y $C$ de ajuste de la función parabólica del caudal medio coinciden exactamente con los promedios de los coeficientes $A_{i}, B_{i}$ y $C_{i}$ de ajuste de la función de predicción del caudal de cada emisor individual.

d) El coeficiente de variación de fabricación $C V$ en las dos formas propuestas (ensayo y predicho) varía con la presión, tanto para emisores no autocompensantes como autocompensantes. 
e) Para los emisores no autocompensantes, las variaciones del $C V_{\text {ensayo }}$ y del $C V_{\text {predicho }}$ con la presión pueden predecirse mediante funciones de ajuste tipo potencial.

f) Para los emisores autocompensantes, las variaciones del $C V_{\text {ensayo }}$ y del $C V_{\text {predicho }}$ con la presión pueden predecirse mediante funciones de ajuste tipo parabólico.

g) Los valores de $\overline{C V}_{\text {continuo }}, \overline{C V}_{\text {discreto }}$ y $C V_{9261}$ calculados a partir de los caudales realmente medidos en el ensayo difieren poco de los obtenidos a partir de los caudales predichos, por lo que los modelos de predicción tanto de los caudales como del $C V$ es perfectamente válido.

h) El $C V_{9261}$ difiere en general de los $\overline{C V}_{\text {continuo }}$ y $\overline{C V}_{\text {discreto }}$ y no se considera un indicador apropiado para caracterizar un emisor, especialmente si es de tipo autocompensante.

i) La influencia de la presencia de datos anómalos se minimiza si se utilizan los caudales predichos para cada emisor individual.

Por todas ellas se recomienda utilizar el modelo propuesto en este trabajo para determinar el coeficiente de variación de un emisor CV.

\section{Bibliografía}

AENOR (2010). UNE-EN ISO 9261:2010: Equipos de riego. Emisores y tuberías emisoras. Especificaciones y métodos de ensayo. (2010).

AENOR (2012). UNE 53367-1:2012: Plásticos. Sistemas de canalización en materiales plásticos para conducción de agua para microirrigación. Polietileno (PE). Parte 1: Especificaciones para tubos. (2012).

Bralts, V. F., Wu, J. P., \& Gitlin, H. M. (1981). Manufacturing variation and drip irrigation uniformity. Transactions of the ASAE, 24

Demir, V., Yurdem, H., \& Degirmencioglu, A. (2007). Development of prediction models for friction losses in drip irrigation laterals equipped with integrated in-line and on-line emitters using dimensional analysis. Biosystems Engineering, 96(4), 617-631.

Karmeli, D., \& Keller, J. (1975). Trickle irrigation design. Glendora, California (USA): Rain Bird Sprinkler Manufacturing Corporation.

Keller, J., \& Karmeli, D. (1974). Trickle irrigation design parameters. Transactions of the ASAE, 17

Rodrigo, J., Hernández, J. M., Pérez, A., \& González, J. F. (1997). Riego localizado. Madrid: Mundi-Prensa: Ministerio de Agricultura, Pesca y Alimentación.

Royuela, Á, \& Turégano, J. V. (2012). Assessment of hydraulic behaviour of regulated emitters in laboratory. Actas De La International Conference of Agricultural Engineering. CIGR-AgEng2012. Valencia

Solomon, K. H. (1979). Manufacturing variation of trickle emitters [irrigation equipment]. Transactions of the ASAE, 22

Turégano, J. V. (2014). Modelización del comportamiento hidráulico de una subunidad de riego localizado. Riunet: Repositorio Institucional de la UPV. 